\title{
Tunable axial bursts using annularly distributed phase masks
}

\author{
Jorge Ojeda-Castaneda, ${ }^{1}$ Sergio Ledesma, ${ }^{1}$ and Cristina M. Gómez-Sarabia ${ }^{2}$ \\ ${ }^{1}$ Electronics Department, University of Guanajuato, Salamanca 36885, México \\ ${ }^{2}$ Digital Arts Department, University of Guanajuato, Salamanca 36885, México
}

Received December 10, 2012; accepted December 28, 2012; published December 31, 2012

\begin{abstract}
We identify a family set of fractional power, annularly distributed phase-masks for generating axial irradiance bursts. We show that by changing the phase delay, for certain fractional power, one can generate either short tail or long tail axial irradiance bursts. To this end, we discuss the use of a pair of angularly modulated, Alvarez-Lohmann lens.
\end{abstract}

For optical alignment, micro machining and confocal scanning it is desirable to shape the point spread function of an optical system. Specifically, for engineering the axial irradiance distribution it is convenient to exploit the McCutchen theorem, which relates through a Fourier transformation the axial complex amplitude distribution with the angular average of the generalized pupil function [1-12].

Here we apply the McCutchen theorem for identifying a family set of radial phase masks which generate axial irradiance distributions that generate axial irradiance bursts. We show that, for a fixed fractional power, one can control the axial irradiance burst by using an angularly modulated Alvarez-Lohmann lens [13-19].

As part of our proposal, we revisit briefly the definition of the Strehl ratio vs. focus errors. Along our discussion, we present a new pair of an Alvarez-Lohmann lens. Then, we identify certain annularly distributed phase masks. Finally, we show numerical evaluations of either short tail axial bursts or long tail axial bursts.

In Fig. 1 we show the schematics of the optical system under discussion. The complex amplitude transmittance of the radial phase mask is

$$
R(\rho, \phi)=\mathrm{e}^{i 2 \pi(a \phi) F(\rho)} \operatorname{circ}\left(\frac{\rho}{\Omega}\right) .
$$

In Eq. (1), we use Greek letters for denoting the polar coordinates in the pupil aperture $(\rho, \phi)$. The letter $\rho$ is the radial spatial frequency whose maximum value is the cutoff spatial frequency $\Omega$. Consequently, the circ function represents the circular pupil aperture with radius $\Omega$. The letter $\phi$ is the polar angle on the circular pupil aperture. The lower case letter "a" denotes the optical path difference; and the function $F(\rho)$ is a real annularly distributed function, to be specified in what follows.

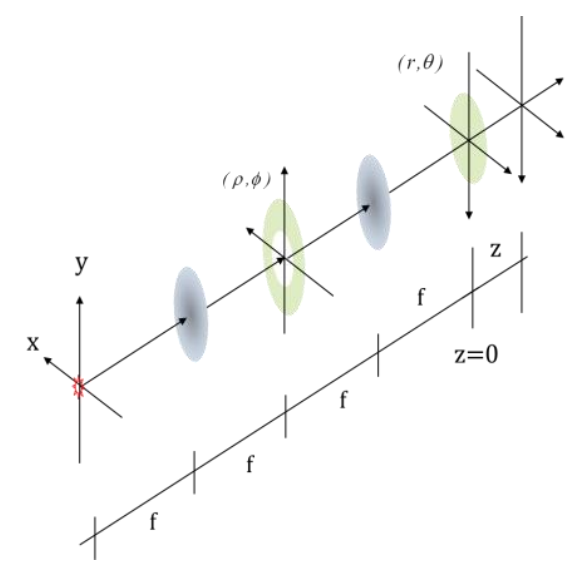

Fig. 1. Schematics of the telecentric optical setup.

Now, following the proposal of the Alvarez-Lohmann lens, we apply Eq. (1) to form the annularly distributed pair:

$$
Q(\rho ; \beta)=R\left(\rho, \phi+\frac{\beta}{2}\right) R^{*}\left(\rho, \phi-\frac{\beta}{2}\right) .
$$

In Eq. (2) the Greek letter $\beta$ denotes the value of the inplane rotation angle, which is introduced between the elements of the pair. By substituting Eq. (1) in Eq. (2), we have that

$$
Q(\rho ; \beta)=\mathrm{e}^{i 2 \pi(a \beta) F(\rho)} \operatorname{circ}\left(\frac{\rho}{\Omega}\right) .
$$

It is apparent from Eq. (3) that the value of $\beta$ controls the total optical path difference of the annularly distributed pair. Within the paraxial regime, we recognize that the generalized pupil is

$$
P(\rho, \phi ; \beta)=Q(\rho ; \beta) \mathrm{e}^{-i \pi \lambda z \rho^{2}} \operatorname{circ}\left(\frac{\rho}{\Omega}\right) .
$$

In Eq. (4) the value of $z$ specifies the axial distance between the in-focus plane and the detection plane; as 
depicted in Fig. 1. The complex amplitude distribution of the amplitude point spread function is

$$
p(r, \theta, z ; \beta)=\int_{0}^{\Omega} \int_{0}^{2 \pi} Q(\rho ; \beta) \mathrm{e}^{-i \pi \lambda z \rho^{2}+i 2 \pi r \rho \cos (\theta-\phi)} \rho d \rho d \phi
$$

We set $r=0$ in Eq. (5) and we use the following variables:

$$
\begin{aligned}
W=-\frac{\lambda z \Omega^{2}}{2}, \quad \zeta & =\left(\frac{\rho}{\Omega}\right)^{2}-\frac{1}{2}, \quad G(\zeta)=F(\rho), \\
q(W) & =p(0, \theta, z ; \beta) .
\end{aligned}
$$

In Eq. (6) the letter $W$ denotes the focus error coefficient in units of wavelength. The normalized version of the axial irradiance distribution is

$$
s(W)=\frac{|q(W)|^{2}}{|q(0)|^{2}} .
$$

The function $s(W)$ is also known as the Strehl ratio vs. focus error. From Eqs. (6)-(7) we obtain:

$$
s(W)=\frac{\left|\int_{-\frac{1}{2}}^{\frac{1}{2}} \mathrm{e}^{i 2 \pi(a \beta) G(\zeta)} \mathrm{e}^{i 2 \pi W \zeta} \mathrm{d} \zeta\right|^{2}}{\left|\int_{-\frac{1}{2}}^{\frac{1}{2}} \mathrm{e}^{i 2 \pi(a \beta) G(\zeta)} \mathrm{d} \zeta\right|^{2}} .
$$

Next, for the function $G(\zeta)$ in Eq. (8), we propose to employ a generalized version of the pupil masks in references [20, 21]; namely

$$
G(\zeta)=\operatorname{sgn}(\zeta)|\zeta|^{t}
$$

In Eq. (9) the sign function is denoted as $\operatorname{sgn}(\zeta)$. And the Latin letter " $t$ " describes a positive real number, which represents the fractional power of the phase delay. In Fig. 2 we display the function $G(\zeta)$ for $0 \leq t \leq 15$.

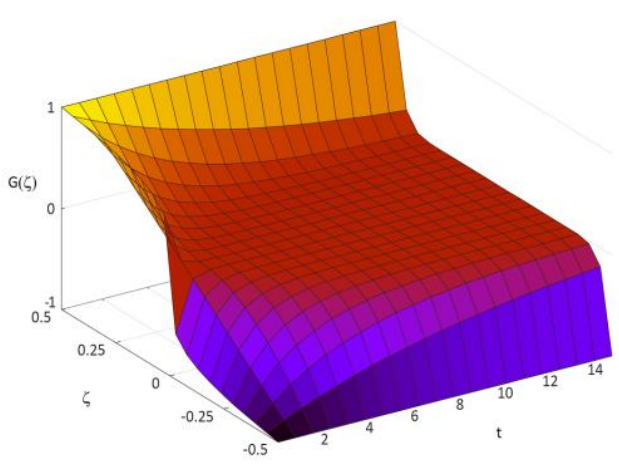

Fig. 2. Phase variation $G(\zeta)$ for fractional power wavefronts.

Next, we employ the definitions in Eq. (6) and Eq. (8) for identifying the annularly distributed phase variations

$$
F(\rho)=\operatorname{sgn}\left(\rho-\frac{\Omega}{\sqrt{2}}\right)\left|\left(\frac{\rho}{\Omega}\right)^{2}-\frac{1}{2}\right|^{t} .
$$

One member of this family is already reported in reference [23]. In Fig. 3, we depict the function $F(\rho)$ for $0 \leq t \leq 15$.

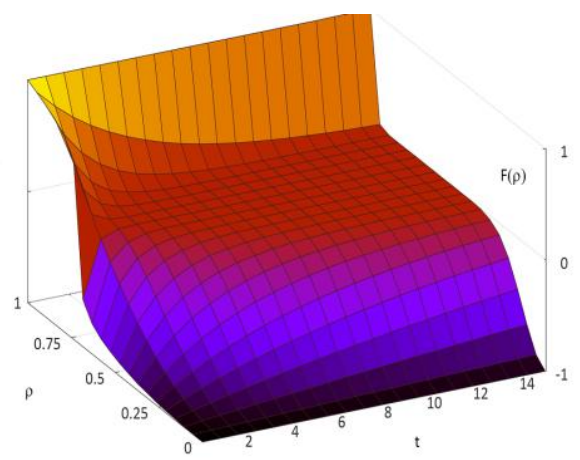

Fig. 3. Annularly distributed phase variation $F(\rho)$.

For numerically evaluating the Strehl ratio in Eq. (8), we perform a fast Fourier transform, as described in reference [22]. Our program uses 1024 points and is written in $\mathrm{C}++$ language. The program includes a set of Graphic User Interface (GUI) elements for manipulating the parameters "a" and " $t$ ". We find numerically that by setting the fractional power $t=2.81 \pm 0.34$ and for an optical path difference $a=0.786 \pm 0.018$, the axial irradiance distribution exhibits a short tail burst; as depicted in Fig. 4. 


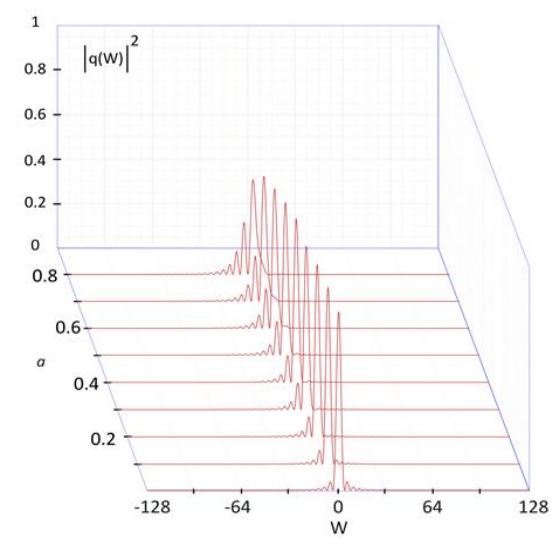

Fig. 4. Strehl ratio for an axial short tail burst, if $t=3$.

It is apparent from Fig. 4 that for the zero optical path difference, $a=0$, the axial irradiance distribution is the well-known irradiance distribution

$$
s(W)=\left[\frac{\sin (\pi W)}{(\pi W)}\right]^{2}
$$

However, as the optical path difference approaches $a=0.8$ the axial irradiance distribution becomes asymmetrical. There is a short tail, axial burst. We note that it is a good choice to set $t=3$, as in reference [23]. Furthermore, from Eq. (3), we recognize that it is physically feasible to achieve the optical path difference requirement, $a \beta=0.8$.

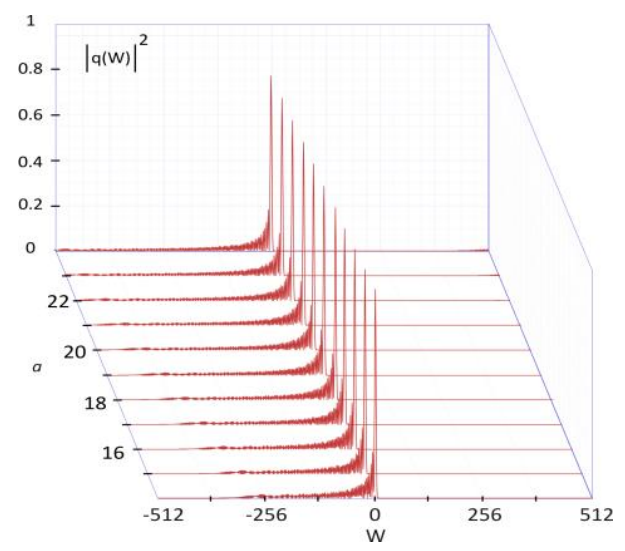

Fig. 5. Strehl ratio for an axial long tail burst, if $t=6$.

Finally, we find numerically that by setting the fractional power $t=7.5 \pm 2.5$ and for an optical path difference $a=27.5 \pm 2.5$, the axial irradiance distribution exhibits a long tail burst; as is depicted in Fig. 5. In this later graph the range of $\mathrm{W}$ is larger than the one in Fig. 4. Of course, as before, the axial burst disappear for $a=0$.
Summarizing, we have identified a family set of annularly distributed phase masks that generate axial irradiance bursts. We have noted that some members of the family set are able to generate a short tail burst; while another set of members have long tails. We have presented the use of two angular phase masks for controlling an axial irradiance burst.

We express our gratitude to CoNaCyT, México, for financial support, through the research grant 157276; as well as to PROMEP for the grants 103.5/10/4612 and PTC-197 D.

\section{References}

[1] C.W. McCutchen, J. Opt. Soc. Am. 54, 240-244 (1964).

[2] C.W. McCutchen, J. Opt. Soc. Am. A 8, 868-870 (1991).

[3] C.W. McCutchen, J. Opt. Soc. Am. A 19, 1721-1721 (2002).

[4] J. Ojeda-Castaneda, P. Andrés, A. Díaz, Opt. Lett. 11, 487-489 (1986).

[5] J. Ojeda-Castaneda, L.R. Berriel-Valdos, E. Montes, Appl. Opt. 26, 2770-2772 (1987)

[6] J. Ojeda-Castaneda, P. Andrés, A. Díaz, J. Opt. Soc. Am. A 5, 12331236 (1988).

[7] J. Ojeda-Castañeda, A. Díaz, App. Opt. 27, 4163-4165 (1988).

[8] A. Kołodziejczyk, S. Bara, Z. Jaroszewicz, M. Sypek, J. Mod. Opt. 37, 1283-1286 (1990).

[9] J. Ojeda-Castañeda, M. Martínez-Corral, P. Andrés, Appl. Opt. 31, 4600-4602 (1992).

[10] J. Sochacki, S. Bara, Z. Jaroszewicz, A. Kołodziejczyk, Opt. Lett. 17, 79 (1992).

[11] J. Ojeda-Castañeda, G. Ramírez, Opt. Lett. 18, 87-89 (1993).

[12] J. Ojeda-Castañeda, M. Martínez-Corral, P. Andrés and A. Pons, Appl. Opt. 33, 7611-7616 (1994).

[13] A.W. Lohmann, Italian patent 727, 848 (June 19, 1964).

[14] L.W. Alvarez, U.S. patent 3, 305, 294 (December 3, 1964).

[15] A.W. Lohmann, British patent 998, 191 (May 29, 1965).

[16] A.W. Lohmann, D.P. Paris, Appl. Opt. 6, 1567-1570 (1967).

[17] I.A. Palusinski, J.M. Sasian, J.E. Greivenkamp, Appl. Opt. 38, 86-90 (1999).

[18] G. Mikuła, Z. Jaroszewicz, A. Kolodziejczyk, K. Petelczyc, M. Sypek Opt. Exp. 15, 9184-9193 (2007)

[19] J. Ojeda-Castaneda, J.E.A. Landgrave, C.M. Gómez-Sarabia, Appl. Opt. 47, E99-E105 (2008).

[20] A. Sauceda, J. Ojeda-Castaneda, Opt. Lett. 29, 560-562 (2004).

[21] A. Castro, J. Ojeda-Castaneda, Appl. Opt. 43, 3474-3479 (2004).

[22] N. Brenner, C. Rader, IEEE Acoustics, Speech Signal Proc. 24, 264 266 (1976).

[23] J. Ojeda-Castañeda, J.E.A. Landgrave, H.M. Escamilla, Opt. Lett. 30, 1647-1649 (2005). 